\section{Análise Preditiva da Densidade Mineral Óssea em Adolescentes Brasileiros Eutróficos do Sexo Masculino}

\section{RESUMO}

Considerada um grave problema de saúde pública, estudos indicam que a osteoporose é uma doença pediátrica com manifestações clínicas apresentadas na senilidade. O diagnóstico de osteopenia/ osteoporose é normalmente feito na prática clínica através da densitometria óssea por atenuação de raio-x de dupla energia (DXA). $O$ exame é de alto custo e de difícil aplicação em populações pediátricas em virtude do emprego de aparelhagem específica e software especial. O presente estudo propõe equações preditivas para avaliação da densidade mineral óssea (DMO) em adolescentes do sexo masculino. Foram recrutados 61 adolescentes saudáveis na faixa de 10 a 19 anos e obtidos a idade óssea (através do raio-x de mão e punho esquerdo), maturação sexual, o peso, a estatura, a DMO na coluna lombar e no fêmur proximal total. A estruturação dos modelos de predição foi realizada pela análise de regressão múltipla "stepwise". Os resultados indicaram modelos de predição para DMO com coeficientes de correlação de 0,87 para região da coluna e 0,80 para região femoral. Esses resultados confirmam que a DMO depende da idade esquelética e do crescimento físico durante a adolescência. As equações propostas são de fácil aplicação, baixo custo operacional e com poder explicativo superior a $75 \%$ e $65 \%$, respectivamente para a DMO da coluna lombar e fêmur proximal total. (Arq Bras Endocrinol Metab 2006;50/1:105-113)

Descritores: Equações preditivas; Densidade mineral óssea; Adolescentes; Massa óssea

\section{ABSTRACT}

\section{A Predictive Analysis from Bone Mineral Density Among Eutrophic Brazilian Male Adolescents.}

Osteoporosis is considered one of the most serious public health diseases and studies show that it is a pediatric disease that manifests during senility. It is usually diagnosed in clinical practice by dual-energy $\mathrm{x}$-ray absorptiometry (DXA). This exam has a high cost and it is difficult to apply to a pediatric population because specific equipment and special software are required. This study presents predictive equations to evaluate the bone mineral density (BMD) among male adolescents. To better predict the equations, some parameters were used as the bone age (by left hand and wrist x-ray), pubertal stages, weight, height and the lumbar spine and femur BMD from 61 healthy adolescents, ages 10 through 19 years. The prediction models were carried through analysis of multiple regressions stepwise. The results have indicated a prediction model for BMD with a coefficient of 0.87 and 0.80 , respectively for the spine and the femoral regions. In conclusion, the results have confirmed that BMD depends on the skeletal age and the physical growth during adolescence. The proposed equations are easy to use, has a low operational cost, and the clarification rate is $75 \%$ and $65 \%$ for lumbar spine and femoral BMD, respectively. (Arq Bras Endocrinol Metab 2006;50/1:105-113) artigo original

\author{
Carla C. Silva \\ Tamara B.L. Goldberg \\ Altamir S. Teixeira \\ José C. Dalmas
}

Faculdade de Medicina de Botucatu - UNESP (CCS, TBLG, AST) e Universidade Estadual de Londrina - UEL (JCD)

Recebido em 13/12/04

Revisado em 09/05/05 e 13/09/05

Aceito em 05/12/05 
Keywords: Predictive equation; Bone mineral density; Adolescents; Bone mass

D URANTE A ADOLESCÊNCIA ocorre grande expansão da massa óssea em função do estirão de crescimento e da alta taxa de mineralização, que se evidenciam nesse período da vida. Sabe-se que grande quantidade do capital mineral ósseo presente na vida adulta foi incorporado durante a fase de crescimento físico da infância e adolescência $(1,2)$. Assim, a quantidade de massa óssea ganha até o final da adolescência, mas principalmente dos 14 aos 16 anos, é um dos fatores determinantes para a proteção da osteopenia e posteriormente da osteoporose, na vida futura (3-5).

Segundo vários autores assinalam, um número considerável de doenças ou tratamentos diversificados nessa fase crítica de incorporação da massa óssea podem redundar em aquisição inadequada, sendo essa responsável por transtornos que resultem em maior risco para fraturas atuais ou comprometimentos futuros $(6,7)$.

O acompanhamento da saúde óssea, seja individualmente ou em estudos populacionais, tem se tornado um tema de grande interesse, uma vez que a não adequação de ganho de massa óssea pode resultar em graves problemas de saúde pública, com grande impacto econômico e social. Para que ocorra uma adequação da mineralização óssea, três prováveis fatores devem se inter-relacionar: os níveis de hormônios circulantes que agem no processo de calcificação, a sobrecarga mecânica imposta ao esqueleto, além da ingestão adequada de cálcio e vitamina $\mathrm{D}$ e sua produção $(8,9)$.

De acordo com o Consenso Brasileiro de Osteoporose (10), depreende-se que o risco de ocorrência dessa doença pode ser reduzido se houver uma preocupação em aumentar a massa óssea durante a adolescência e posteriormente, um cuidado com a diminuição na taxa de perda óssea pós-pubertária (10). Neste sentido, o pico de massa óssea e os locais específicos para a avaliação da densidade mineral óssea são indicadores importantes quando se pretende atuar no sentido preventivo a um estado de osteopenia e/ou osteoporose precoce. As principais manifestações clínicas da osteoporose são as fraturas, sendo os locais mais suscetíveis as vértebras, o fềmur proximal e o antebraço $(8,10)$.

Toda essa preocupação se fundamenta na busca da prevenção primária da osteoporose e na tentativa de se reduzir ao máximo os riscos de desenvolvê-la, buscando principalmente maximizar o incremento deste tecido particularmente durante os anos de crescimento físico (4).
A confirmação do diagnóstico de osteopenia/ osteoporose é comumente efetuada na prática clínica, através do exame de densitometria óssea por atenuação de raio-x de dupla energia (DXA). Além de ser um exame de alto custo, sua aplicação em populações pediátricas requer o emprego de um software especificamente desenvolvido para avaliar crianças e adolescentes, o que em muitos serviços torna-se um fator impeditivo à sua aplicabilidade.

Instigados por essa problemática, o presente estudo propõe equações preditivas para avaliação da densidade mineral óssea em adolescentes do sexo masculino. Buscou-se mediante avaliação de indicadores de crescimento físico, maturação óssea e sexual, apresentar o conjunto de variáveis com o mais alto coeficiente de explicação para a densidade mineral óssea.

Com o intuito de contribuir para o avanço nos conhecimentos relativos à saúde óssea em adolescentes, na prevenção de danos futuros e proporcionar indicativos para uma avaliação hipotética com menores custos e de fácil aplicabilidade na prática clínica, esse estudo foi proposto. Entretanto, ressalta-se que os resultados encontrados, uma vez advindos de uma população específica e com características próprias, apenas balizam a compreensão dos fatores envolvidos na mineralização óssea, não sendo portanto considerados substitutos das técnicas altamente sensíveis utilizadas para avaliação da densidade mineral óssea.

\section{MÉTODOS}

Participaram do presente estudo 61 adolescentes do sexo masculino de 10 a 19 anos, voluntários, saudáveis e estudantes de colégios da rede particular de ensino, situados no município de Botucatu, Estado de São Paulo. A pesquisa teve aprovação da Comissão de Ética em Pesquisa da Faculdade de Medicina de Botucatu UNESP, e os adolescentes e seus responsáveis tiveram ciência do seu conteúdo, através de explanações realizadas no ambiente escolar. Para que participassem da pesquisa, receberam e devolveram o termo de consentimento livre e esclarecido assinado pelo binômio adolescente e seu responsável.

Os critérios de inclusão exigiam que os adolescentes estivessem com peso corporal entre os percentis 10 e 90 e estatura entre os percentis 10 e 97,5 para cada faixa de idade (11) e com índice de massa corporal (IMC) adequado para a idade (12) e sexo masculino para os respectivos pontos de corte propostos pelo Centers for Disease Control and Prevention, que são: eutrofia, IMC entre os percentis 5 e 85 ; sobre- 
peso, entre os percentis 85 e 95 ; obesidade, percentil maior que 95 (13). Portanto, só foram incluídos aqueles considerados eutróficos.

Como critérios de exclusão determinou-se que não participariam do estudo: os adolescentes com história de prematuridade ou baixo peso ao nascimento; aqueles que estivessem vinculados a qualquer modalidade esportiva, a exceção das aulas de Educação Física do próprio colégio, os submetidos à terapia prolongada com corticóides ou que utilizassem suplementação com cálcio e/ou ferro nos últimos doze meses que antecediam a pesquisa. Também foram excluídos adolescentes que apresentassem as seguintes doenças: diabetes mellitus, desnutrição aguda ou crônica, doenças ósseas congênitas ou adquiridas, doenças gastrointestinais acompanhadas de máabsorção, história de nefropatia, com ou sem insuficiência renal crônica, endocrinopatias, puberdade precoce ou atrasada, consumo crônico de drogas, fibrose cística, doença celíaca, utilização de drogas que afetem o metabolismo ósseo negativamente, como anticonvulsivantes, antiácidos com alumínio. Quanto à avaliação dietética, realizada pela utilização de registro alimentar de três dias (14) e quantificação centesimal dos inquéritos alimentares mediante utilização de um sistema computacional de análise nutricional (15), foram excluídos os adolescentes que fizessem uso exclusivo de dieta vegetariana, aqueles com alto consumo de fibras, cafeína ou refrigerantes, os que não consumiam produtos lácteos diariamente, aqueles etilistas, bem como os tabagistas.

Quanto à questão étnica apontada em pesquisas internacionais (16) como fator determinante no ganho da massa óssea, assumiu-se que a população brasileira apresenta grande miscigenação, entretanto salienta-se que, entre os adolescentes avaliados, nenhum era de cor negra ou filho de casal afro-descendente ou de pais de origem exclusivamente oriental.

Dos 497 escolares do sexo masculino matriculados nos colégios, 61 atenderam aos critérios de inclusão e consentiram em participar de todo o processo de coleta de dados. Para a obtenção do número de adolescentes incluídos no estudo, foi realizado o cálculo amostral. Admitiu-se nível de significância de $5 \%$ para o intervalo de confiança de $95 \%$, determinados para uma distribuição normal, com $\mathrm{Z}=1,96$ (17). Nesse processo, como não era conhecido o desviopadrão populacional, foi necessário estimá-lo através de uma amostra retirada da população em questão. Assim, considerando um erro máximo (E) que foi admitido em torno do valor médio estimado, o tamanho da amostra foi determinado mediante a utilização de recurso matemático, sendo este modelo avaliado e validado (18).

Os adolescentes que se enquadravam aos critérios de elegibilidade foram então convidados junto com seus responsáveis a comparecerem ao Ambulatório de Adolescentes do Hospital das Clínicas da Faculdade de Medicina de Botucatu - UNESP, onde foi realizada uma entrevista com seus responsáveis, seguida de exame físico geral e especial, para que qualquer alteração física fosse detectada. Foram avaliados os caracteres sexuais secundários por ocasião do exame físico e o resultado foi confrontado aos critérios de Tanner (19). A idade óssea foi obtida mediante a avaliação do grau de maturação esquelética. $\mathrm{O}$ método utilizado foi o de Greulich \& Pyle (1959), chamado de método GP, em que se faz a radiografia de mão e punho esquerdo a ser posteriormente comparada ao Atlas (20).

O exame para determinação do conteúdo mineral ósseo (CMO) em gramas (g) e da densidade mineral óssea (DMO), expressa em $\mathrm{g} / \mathrm{cm}^{2}$ de cada adolescente foi realizado através de uma unidade de densitometria óssea por atenuação de raio-x de dupla energia (DXA), utilizando um aparelho Hologic QDR 2000-Plus com a inserção de software pediátrico para adequada avaliação da massa óssea. As regiões da coluna lombar entre Ll-L4 e do fêmur proximal total, incluindo as regiões: cólo do fêmur, região trocantérica, intertrocantérica e área de Ward, foram avaliadas. Quanto à determinação do coeficiente de variação (CV) para cada uma das áreas examinadas, este valor foi calculado entre momentos distintos, a partir da reprodução do exame, para observação de eventuais variações de precisão que poderiam ocorrer. É importante ressaltar que a realização de todos os exames ocorreu em condições apropriadas, dentro do controle técnico de qualidade (21). De posse destes resultados obteve-se o CV para coluna lombar de $0,6 \%$ e região do fêmur proximal total de 1,1\%, mantendo-se arquivados os coeficientes através de documentação. Os coeficientes encontrados se enquadram dentro de critérios de precisão para análise da $\mathrm{DMO} \mathrm{em}$ ambos os sítios avaliados, que de acordo com Khan e cols. (2001) podem variar de 0,5 a $1,5 \%$ na coluna lombar e 1 a $2 \%$ no fêmur proximal total (22). Esse método de avaliação da massa óssea propicia uma análise altamente precisa e com baixa exposição à radiação, sendo adequado para crianças e adolescentes (23-25).

O tratamento estatístico dos dados foi realizado através do pacote computadorizado Statistical Package for Social Sciences (SPSS) versão 7.5. Num primeiro momento foram calculados os coeficientes de 
correlação linear simples de Pearson entre as variáveis da massa óssea e os aspectos morfológicos e pubertários dos adolescentes. Num segundo momento, utilizando-se recursos da análise de regressão múltipla "stepwise" e tendo como variável dependente a DMO, esta foi confrontada com as variáveis independentes. A escolha das equações elaboradas pelo modelo foi realizada segundo os seguintes critérios: a) nível de significância dos modelos; b) maior coeficiente de correlação múltipla (R); c) maior coeficiente de determinação $\left(\mathrm{r}^{2}\right)$; d) praticidade na utilização do modelo preditivo.

\section{RESULTADOS}

Num primeiro momento, são apresentadas as características gerais da população de adolescentes estudados com o objetivo de compreender o processo de mineralização óssea desta fase específica da vida. Para tanto, a faixa etária de 10 a 19 anos foi agrupada da seguinte forma: 10 anos a 12,0 anos completos (Faixa Etária 1 ou FEl); 12 anos e um dia a 14,0 anos completos (FE2); 14 anos e um dia a 16,0 anos completos (FE3) e de 16 anos e um dia a 19,0 anos completos (FE4).
A tabela 1 indica os valores médios e desviospadrão entre os grupos de idade com relação aos indicadores de crescimento, peso e estatura, IMC, ingestão de cálcio (mg/dia), maturação óssea, representada pela evolução na idade óssea, e indicadores de massa óssea, CMO e a DMO tanto para coluna lombar (LlL4) como para as regiões do fêmur proximal total.

Os indicadores da massa óssea aumentam com o avançar da idade cronológica e maturação óssea, bem como com as alterações evolutivas no peso e estatura, típicas desta fase pubertária. Na tabela 1 é possível observar as diferenças estatisticamente significantes entre os grupos etários e as variáveis avaliadas.

Na tabela 2 estão expressas as variáveis relativas à mineralização óssea de acordo com os estágios pubertários (Gl a G5).

Com relação ao indicador de maturação sexual, os resultados da DMO apontaram uma variação crescente de $0,584 \mathrm{gr} / \mathrm{cm}^{2}$ em Gl (Genitais 1) para $0,989 \mathrm{gr} / \mathrm{cm}^{2}$ em G5 (Genitais 5 ), na coluna lombar. De forma similar no fêmur proximal total, a DMO variou de $0,787 \mathrm{gr} / \mathrm{cm}^{2} \mathrm{em} \mathrm{Gl}$ para $1,109 \mathrm{gr} / \mathrm{cm}^{2} \mathrm{em}$ G5, demonstrando um aumento ascendente e significativo $(\mathrm{p}<0,01)$ em função das alterações provocadas pela maturação biológica.

Tabela 1. Valores médios e desvios-padrão entre os grupos de idade.

\begin{tabular}{|c|c|c|c|c|}
\hline Variáveis & Estrati & cação por Faixc & a Etária & \\
\hline & $\begin{array}{c}\mathrm{FEl}=10-12 \\
(\mathrm{n}=14)\end{array}$ & $\begin{array}{c}\mathrm{FE} 2=>12,1-14,0 \\
(\mathrm{n}=14)\end{array}$ & $\begin{array}{c}\mathrm{FE} 3=>14,1-16,0 \\
(n=16)\end{array}$ & $\begin{array}{c}\text { FE4 }=>16,1-19,0 \\
(n=17)\end{array}$ \\
\hline Idade óssea (anos) & $10,1 \pm 1,4^{a}$ & $12,5 \pm 1,2^{b}$ & $15,5 \pm 1,6^{c}$ & $18,3 \pm 0,8$ \\
\hline Peso $(\mathrm{kg})$ & $35,7 \pm 4,5^{a}$ & $42,9 \pm 8,8^{b}$ & $58,0 \pm 9,5^{c}$ & $65,2 \pm 6,3$ \\
\hline Estatura (m) & $1,43 \pm 0,07^{a}$ & $1,54 \pm 0,09^{b}$ & $1,71 \pm 0,07$ & $1,75 \pm 0,07$ \\
\hline $\mathrm{IMC}\left(\mathrm{kg} / \mathrm{m}^{2}\right)$ & $17,39 \pm 1,43^{a}$ & $17,74 \pm 1,47^{d}$ & $19,97 \pm 1,69^{c}$ & $21,22 \pm 1,83$ \\
\hline Cálcio (mg/dia) & $825,0 \pm 209,9$ & $811,8 \pm 177,9$ & $897,2 \pm 201,9$ & $963,7 \pm 335,6$ \\
\hline CMO(gr) (L1-L4) & $28,71 \pm 3,95^{a}$ & $36,11 \pm 10,89^{b}$ & $51,02 \pm 13,21^{c}$ & $67,01 \pm 14,32$ \\
\hline $\mathrm{DMO}\left(\mathrm{gr} / \mathrm{cm}^{2}\right)(\mathrm{Ll}-\mathrm{L} 4)$ & $0,617 \pm 0,04^{a}$ & $0,70 \pm 0,12^{b}$ & $0,83 \pm 0,12^{c}$ & $1,015 \pm 0,14$ \\
\hline CMO(gr) (Fêmur) & $26,01 \pm 3,69^{a}$ & $31,63 \pm 9,24^{b}$ & $49,29 \pm 11,66^{c}$ & $57,36 \pm 9,30$ \\
\hline $\mathrm{DMO}\left(\mathrm{gr} / \mathrm{cm}^{2}\right)$ (Fêmur) & $0,80 \pm 0,07^{a}$ & $0,83 \pm 0,08^{b}$ & $0,98 \pm 0,15^{c}$ & $1,14 \pm 0,14$ \\
\hline
\end{tabular}

Tabela 2. Conteúdo e densidade mineral óssea de acordo com os estágios de Tanner (genitais)

\begin{tabular}{|c|c|c|c|c|}
\hline \multirow{2}{*}{ Estágios Tanner } & \multicolumn{4}{|c|}{ Indicadores de Massa Óssea } \\
\hline & $\mathrm{CMO}(\mathrm{Ll}-\mathrm{L} 4)$ & $\mathrm{DMO}(\mathrm{L} 1-\mathrm{L} 4)$ & CMO (Fêmur) & DMO (Fêmur) \\
\hline$G 1(n=6)$ & $25,9 \pm 2,8^{a}$ & $0,584 \pm 0,04^{a}$ & $24,5 \pm 3,4^{c}$ & $0,787 \pm 0,08^{c}$ \\
\hline$G 2(n=12)$ & $29,6 \pm 4,2^{a}$ & $0,639 \pm 0,05^{a}$ & $26,4 \pm 3,7 c$ & $0,806 \pm 0,06^{c}$ \\
\hline G3 $(n=8)$ & $36,4 \pm 6,7^{b}$ & $0,694 \pm 0,10^{b}$ & $33,1 \pm 3,9 c$ & $0,842 \pm 0,06^{c}$ \\
\hline$G 4(n=15)$ & $51,5 \pm 13,4^{b}$ & $0,844 \pm 0,12^{b}$ & $50,0 \pm 13,3$ & $0,999 \pm 0,17$ \\
\hline G5 $(n=20)$ & $65,0 \pm 13,5$ & $0,989 \pm 0,13$ & $55,9 \pm 8,9$ & $1,109 \pm 0,15$ \\
\hline Grupo total & $47,1 \pm 18,5$ & $0,806 \pm 0,18$ & $42,5 \pm 15,5$ & $0,956 \pm 0,18$ \\
\hline
\end{tabular}


Para efetivação de um modelo matemático que pudesse predizer a DMO, recorreu-se inicialmente ao cálculo dos coeficientes de correlação linear simples de Pearson entre as variáveis da massa óssea e os aspectos morfológicos e os caracteres sexuais secundários dos adolescentes. Assim, a DMO dos adolescentes foi considerada variável dependente, e as variáveis independentes foram a idade cronológica, a maturação esquelética e sexual, bem como indicadores de crescimento, peso e estatura.

Os resultados demonstram diferenças significativas e positivas para correlação linear simples entre todas as variáveis, com nível de significância menor que $1 \%$.

Uma análise mais acurada dos resultados permite apresentar o IMC, dentre todos os indicadores estudados, como o mais fraco na correlação, com valores de r variando entre 0,60 e 0,74 para CMO da área da coluna e do fềmur proximal total, respectivamente, mesmo sendo a correlação realizada através do cálculo do escore z para o IMC. A maior correlação entre o CMO foi verificada com o peso corporal, sendo que para a coluna lombar o escore foi de $r=0,88$, enquanto que para a região do fêmur proximal total o índice de correlação chegou a $r=0,91$. De forma similar, a análise de correlação mais elevada para a DMO foi com o peso corporal. Para a região da coluna lombar a correlação com o peso foi de $r=0,85$, enquanto que na região do fềmur proximal total a correlação foi de $r=0,80$. Com relação aos indicadores de maturação esquelética e sexual observa-se correlação superior a 0,70 , demonstrando grande participação da maturação biológica frente ao incremento da massa óssea nesses adolescentes.

Na seqüência, recorrendo-se a recursos da análise de regressão múltipla "stepwise", foram desenvolvi-

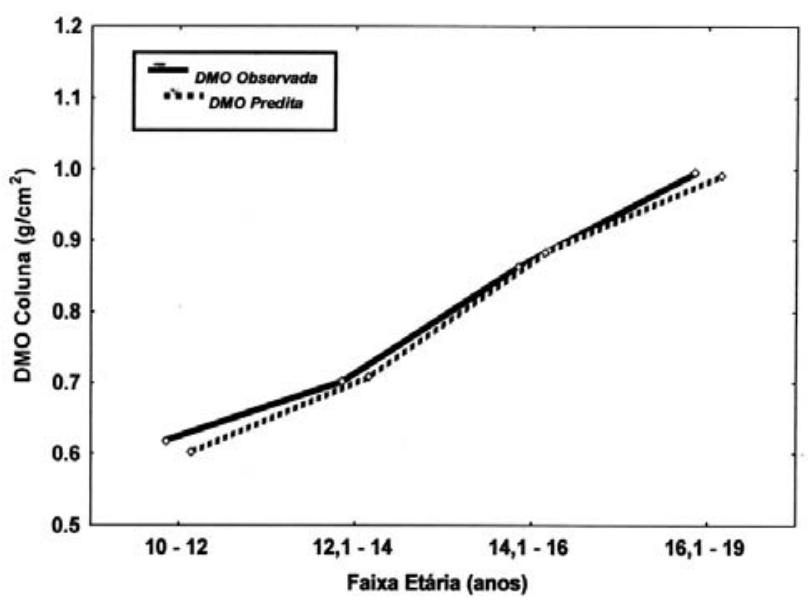

Gráfico 1. Valores observados pela DMO da coluna versus valores preditos. dos modelos matemáticos para a estimativa da DMO em adolescentes eutróficos.

De acordo com a análise de regressão, o melhor modelo preditivo seguindo os critérios supracitados no capítulo de métodos foi:

$$
\begin{aligned}
& D M O_{\text {coluna }}=0,127+0,022959 \times \text { Idade Óssea }+0,006751 \\
& \text { x Peso }
\end{aligned}
$$

Observa-se que a análise de regressão múltipla propôs a combinação das variáveis idade óssea e peso corporal para compor a equação preditiva da DMO da coluna lombar. Em conjunto, estas variáveis demonstraram um coeficiente de determinação próximo a 77\% para a predição, com um $p$ menor que $1 \%$. Salienta-se que o acréscimo de outras variáveis não modificou o coeficiente de determinação encontrado no desenvolvimento do melhor modelo preditivo.

O gráfico 1 , na seqüência, indica os valores observados pelo densitômetro confrontados aos valores previstos pelo modelo preditivo, apresentados ordenadamente de forma crescente à idade dos adolescentes. Observa-se no gráfico que os valores preditos coincidem em vários pontos com os valores observados, indicando que a estimativa do modelo é bastante precisa.

O mesmo procedimento estatístico foi aplicado aos dados obtidos na região do fêmur proximal total. Para esta região, a análise de regressão múltipla indicou o peso corporal como variável mais adequada para explicar a variação na DMO dos adolescentes, com um coeficiente de determinação próximo de $65 \%$ e com um $p$ menor que $1 \%$. O modelo preditivo proposto foi: $D M O_{\text {fémur proximal total }}=0,401741+0,01062 \times$ Peso

No gráfico 2 observa-se o comportamento entre os valores observados e os valores preditos para a esta

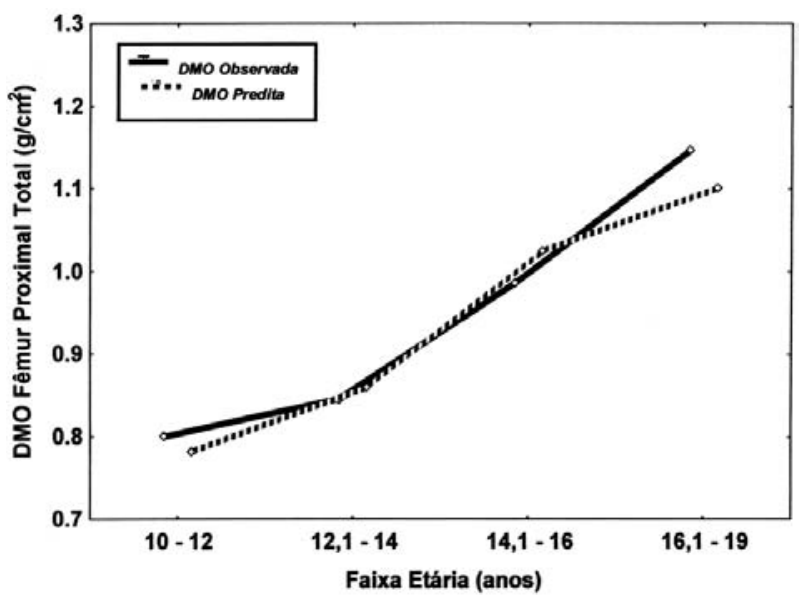

Gráfico 2. Valores observados pela DMO do fêmur versus valores preditos. 
região, ordenados pela idade cronológica dos adolescentes. De forma similar ao comportamento dos valores observados e preditos para a coluna lombar, grande parte dos pontos estimados, pela equação proposta, coincidem com os valores observados pela avaliação obtida pela densitometria óssea.

Nos gráficos que se seguem são demonstrados os comportamentos de normalidade dos resíduos relacionados aos modelos propostos para coluna lombar e para a região do fêmur proximal total, respectivamente (gráficos 3 e 4 ). Os valores residuais indicam a diferença entre os valores previstos pela equação e os valores observados a partir da avaliação pelo densitômetro. A indicação dos pontos próximos à reta demonstram que os dados têm uma distribuição normal, estando os valores calculados próximos aos valores esperados $(-1)$ (0) e $(+1)$. Para ambos os sítios avaliados observa-se que a grande maioria dos pontos aparecem dispostos próximos à reta, indicando normalidade em sua distribuição.

\section{DISCUSSĀO}

A mineralização óssea é um processo multifatorial resultante de vários elementos que atuam modulando a aquisição da massa óssea. Destacam-se os aspectos genéticos, a ingestão adequada de cálcio e vitamina D e a sua produção (26), os níveis de hormônios circulantes que agem no processo de calcificação, as alterações no peso corporal e a sobrecarga mecânica imposta ao esqueleto $(8,9)$ e, durante a segunda década da vida, ainda se incluem as evidentes alterações nas dimensões corporais e hormonais que conduzem o adolescente ao amadurecimento biológico $(3,27)$.

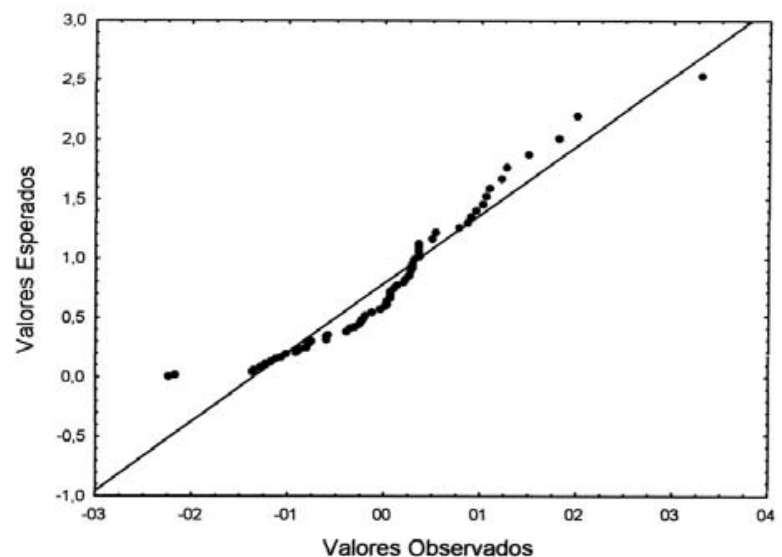

Gráfico 3. Comportamento gráfico de normalidade dos resíduos do modelo proposto para a DMO da coluna lombar (L1-L4).
Os resultados do presente estudo estão de acordo com outras investigações quanto à correlação entre a DMO e as variáveis antropométricas, como peso corporal, estatura e as alterações na maturação óssea e sexual $(2,5,28,29)$.

Em estudo com crianças pré-púberes, entre sete e oito anos, Pessoa e cols. verificaram correlações positivas entre o CMO e a DMO na região da coluna lombar com a idade óssea, peso corporal e estatura. Esses autores sugerem que a interpretação da massa óssea em pré-púberes deve estar atrelada à variação do peso corporal e da idade óssea (23). De forma similar, Klein e cols. observaram correlação significante entre a DMO de corpo total com a idade cronológica e idade óssea em crianças de aproximadamente 10 anos de idade de ambos os sexos (30).

Em concordância, o presente estudo aponta o peso corporal como um dos indicadores de forte correlação com a massa óssea, sendo indicado, pela análise de regressão, como a variável incluída em ambos modelos preditivos propostos para a DMO. Para a idade óssea e DMO, os coeficientes de correlação foram expressivos, com $r=0,84$ e $r=0,74$ para regiões $d a$ coluna lombar e fềmur proximal total, respectivamente.

De forma similar, Rubin e cols. observaram, mediante a análise de regressão múltipla, que a idade, o peso corporal, a estatura e o estágio pubertário, indicados pelos critérios de Tanner, foram as principais variáveis preditoras da massa óssea. Especificamente, no estudo destes pesquisadores, a predição da DMO da coluna lombar ocorreu a partir da combinação dos eventos pubertários com o peso corporal, demonstrando coeficiente de determinação na ordem de $80 \%$. Os próprios autores destacam que a DMO desta região é altamente responsiva às alterações do crescimento

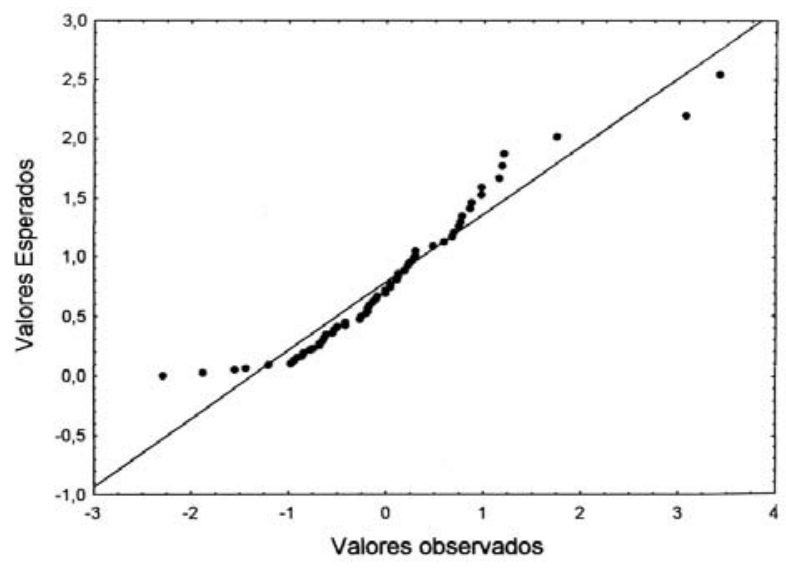

Gráfico 4. Comportamento gráfico de normalidade dos resíduos do modelo proposto para a DMO na região do fêmur proximal total

Arq Bras Endocrinol Metab vol 50 n 1 Fevereiro 2006 
corporal (28). Outros autores também demonstraram participação do peso e dos estágios de Tanner nos modelos desenvolvidos para a densidade óssea de corpo total e de densidade volumétrica da coluna lombar $\left(\mathrm{g} / \mathrm{cm}^{3}\right)$ de meninos e jovens de 4 a 20 anos. No entanto, estes mesmos pesquisadores, desenvolvendo modelos para DMO de coluna lombar (5), ao incluírem apenas peso e estatura, obtiveram um $\mathrm{r}^{2}$ de $85 \%$, e pouco superior, $\mathrm{r}^{2}$ de $88 \%$ quando incluíram peso e ingestão de cálcio. Deve-se ressaltar que os jovens participantes deste estudo eram holandeses, portanto com ingestão média de cálcio próxima à recomendada pelo Dietary Reference Intake (DRI), que é de 1.300 $\mathrm{mg} /$ dia, como já salientado em outros trabalhos desenvolvidos naquele país. Nitidamente, podemos assinalar que nossos jovens apesar de pertencerem a uma classe social privilegiada, apresentavam consumo de cálcio inferior ao recomendado, mas superior ao revelado numa série de trabalhos desenvolvidos em nosso país $(2,31,32)$. Para Jackman e cols. parece evidente que a baixa ingestão desse mineral, durante a fase de crescimento de crianças e adolescentes, resulte em menor mineralização óssea quando comparada à de indivíduos da mesma faixa etária que tiveram ingestão adequada de cálcio (33). Embora o impacto da ingestão de cálcio seja reconhecidamente apresentado como variável importante no processo de mineralização óssea, no presente estudo as variáveis morfológicas indicaram supremacia na determinação da massa óssea, quando determinados os coeficientes de correlação.

Avaliando o sexo feminino, Hannan e cols. propuseram equações preditivas para o CMO e a DMO da coluna lombar (L1-L4) de adolescentes entre 11 e 18 anos. As melhores equações de predição tanto para o CMO como para a DMO incluíam o peso e a estatura das adolescentes. Resultados similares foram observados no presente estudo, onde a análise de regressão incluiu o peso corporal tanto no modelo preditivo da coluna lombar como na região femural. É importante ressaltar que em todos os modelos aqui propostos, a análise de regressão múltipla demonstrou significância estatística $(\mathrm{p}<0,01)$ e coeficientes de correlação, $\mathrm{R}=$ 0,87 e $\mathrm{R}=0,80$ respectivamente, para a coluna lombar e para a região do fềmur proximal total (34).

Para Khan e cols., tanto a região da coluna como a região do fêmur estão expostas ao estresse mecânico gerado pelo peso corporal e à contínua ação da força da gravidade. Na região do fềmur proximal total, a análise de regressão do presente estudo indicou o peso corporal dos adolescentes como a principal variável explicativa, com um coeficiente de determinação próximo a $65 \%$. Este resultado pode ser expli- cado pela absorção de um estresse compressivo entre 3 e 7 vezes o peso corporal, durante a caminhada, sobre a região do quadril (22). Confirmando estes dados, foi evidenciado em um grupo de atletas que participaram de esportes com impacto, tais como basquetebol, ginástica e atletismo, um aumento significativo tanto na DMO da coluna como no fêmur, quando comparados aos atletas que praticavam esportes de carga ativa, sem a imposição da força da gravidade, tais como natação e pólo aquático (24).

Um aspecto crítico relacionado à obtenção do peso corporal seria sua alta variabilidade no decorrer da trajetória da vida. No entanto, no presente estudo, estabeleceu-se como critério de inclusão os adolescentes que estivessem com peso corporal entre os percentis 10 e 90 e estatura entre os percentis 10 e 97,5 , para cada faixa de idade. Esta preocupação esteve atrelada à evidência de que a obesidade poderia se constituir em fator de proteção para fraturas resultantes de uma osteopenia ou osteoporose na senilidade, e que um excesso de peso poderia ser benéfico na aquisição da massa óssea na adolescência $(22,35)$. Acredita-se que estas observações sejam conseqüentes ao "fator" peso corporal exercer uma sobrecarga mecânica importante sobre os sítios ósseos da coluna e do fềmur (36). Entretanto, para a seleção dos adolescentes da amostra, contemplou-se apenas aqueles considerados eutróficos, com o que se excluiu a possibilidade de interferência da condição sobrepeso/ obesidade sobre a aquisição da massa óssea.

Sabe-se que o estresse mecânico ocasionado pelo peso corporal e as contrações musculares aumentam a massa óssea, mas o mecanismo não está completamente esclarecido (37). Carvalho e cols. declaram que o modelamento e remodelamento ósseo pode ser ocasionado por microlesões, estímulos mecânicos, estímulos combinados ou por mecanismos desconhecidos (38). Confirmando a inexistência de consenso na literatura, Klein e cols. não verificaram incremento na massa óssea entre crianças com excesso de peso e aquelas não obesas de mesma idade cronológica (30). De forma similar, Goulding e cols. investigaram o impacto do peso corporal sobre o CMO em 362 crianças e adolescentes de 3 a 19 anos, de ambos os sexos. Os autores estratificaram a amostra em 3 grupos: peso normal, sobrepesos e obesos de acordo com o IMC. Os resultados não apontaram diferenças significantes entre os grupos para o CMO da coluna lombar (L2-L4) (39).

A confrontação dos modelos preditivos do presente estudo com outras investigações foi possível, particularmente com as análises realizadas por Fonseca 
e cols., no Brasil. Nesta investigação, foi efetuada a avaliação do CMO e da DMO na coluna lombar (L2L4) de crianças e adolescentes de 6 a 14 anos de idade. Os autores propuseram modelos preditivos para a avaliação do $\mathrm{CMO}$ e da DMO para esta região, ajustados pela medida da área vertebral, demonstrando ser esta uma variável representativa da variação da massa óssea, na região da coluna lombar de crianças e adolescentes $(29,36)$.

O presente estudo demonstrou, para a casuística específica, coeficiente de determinação similar para a DMO da coluna lombar $\left(\mathrm{r}^{2}=0,76\right)$, quando comparado aos resultados apresentados por Fonseca e cols. $(23,31)$; no entanto, a variação da massa óssea foi observada a partir de medidas antropométricas e maturação esquelética. De forma similar, em publicação recente, Horlick e cols. propuseram vários modelos preditivos para massa óssea de corpo total de crianças e adolescentes entre 6 e 18 anos. Os resultados demonstraram elevados coeficientes de determinação $\left(\mathrm{r}^{2}=0,959\right.$ e $\left.\mathrm{r}^{2}=0,956\right)$ para o CMO do corpo total para crianças e adolescentes do sexo masculino e feminino respectivamente (16). Em concordância com o presente estudo, as variáveis independentes: idade, peso, estatura e etnia foram inseridas no modelo proposto pelos autores, confirmando, assim, o impacto do processo do crescimento físico ocorrido durante este período etário, bem como a imposição étnica sobre a aquisição da massa óssea em crianças e adolescentes.

\section{CONSIDERAÇÕES FINAIS}

A interpretação de dados referentes à massa óssea na população pediátrica é complexa em função das alterações simultâneas da densidade mineral, das dimensões ósseas e das alterações corporais. Os resultados do presente estudo suportam a idéia de que as correlações entre a DMO e as variáveis antropométricas ocorrem em grande parte devido ao aumento das dimensões ósseas durante o processo de crescimento.

Principalmente no que tange às equações aqui propostas, é importante ressaltar que estas não devem ser utilizadas indiscriminadamente, pois os modelos aqui apresentados foram baseados em população saudável de adolescentes do sexo masculino. Portanto, a utilização destas equações em populações pediátricas, que tenham sua saúde óssea comprometida direta ou indiretamente, deve ser observada com cautela, uma vez que os modelos apresentados, apesar de terem alto poder explicativo, deverão ser utilizados em populações com critérios similares de elegibilidade. Além disso, investigações desta natureza não objetivam substituir o exame densitométrico pela utilização de modelos preditivos, mas apenas indicar as variáveis que, isoladas e/ou associadas, possam revelar variações da massa óssea durante o período de crescimento físico da criança e do adolescente, no sentido de contribuir na compreensão do processo de mineralização óssea.

\section{AGRADECIMENTOS}

Apoio da Fundação de Amparo à Pesquisa do Estado de São Paulo - FAPESP (Processo 04/07007-1). A Ilana Goldberg, pela versão do resumo.

\section{REFERÊNCIAS}

1. Campos LMA, Liphaus BL, Silva CAA, Pereira RMR. Osteoporose na infância e adolescência. J Ped 2003;79(6):481-8.

2. Vargas DM, Rigotti T, Gütz CNRM, Lobe MCS, Fernandes JA. Mineralização óssea em crianças e adolescentes com diabetes melito tipo 1. J Ped 2003;79(3):253-8.

3. Silva CC, Goldberg TBL, Teixeira AS, Dalmas JC. Mineralização óssea em adolescentes do sexo masculino: anos críticos para a aquisição da massa óssea. J Ped 2004;6(80):461-7.

4. Bonjour JP, Theintz G, Buchs B, Slosman D, Rizzoli R. Critical years and stages of puberty for spinal and femoral bone mass accumulation during adolescence. J Clin Endocrinol Metab 1991;73:555-63.

5. Boot AM, Ridder MAJ, Pols HAPP, Krenning EP, Muinck Keizer-Schrama SMPF. Bone Mineral density in children and adolescents: Relation to puberty, calcium Intake, and physical activity, J Clin Endocrinol Metab 1997:82:57-62.

6. Kaste SC. Bone mineral density deficits from childhood cancer and its therapy. A review of at-risk patient cohort and available imaging methods. Pediatr Radiol 2004;34:373-8.

7. Lazaretti-Castro M. Por que medir densidade mineral óssea em crianças e adolescentes? J Pediatr 2004;80(6):439-40.

8. Carrascosa A, Guissinyé M. Crescimento e mineralização do esqueleto durante a puberdade e adolescência: Regulação nutricional e hormonal. Anais Nestlé 1998:55:9-17

9. Heaney RP. Calcium, dairy products and osteoporosis. J Am Coll Nutr 2000; 19(suppl):83-99.

10. Neto AMP, et al. Consenso brasileiro de osteoporose 2002. Rev Bras Reumatol 2002;42:343-54.

11. Hamill PVV, Drizd TA, Johnson CL, Reed RB, Roche AF, Moore WM. Physical growth: National Center for Health Statistics Percentiles. Am J Clin Nutr 1979;32:607-29.

12. Prevalence of overweight among adolescents-United States, 1988-91. MMWR Morb Wkly Rep 1994;43:818-21. 
13. Centers for Disease Control and Prevention and National Center for Health Statistics. 2000 CDC growth charts: United States (online) Hyaltsville; 2002. Available from: http://www.cdc.gov/growthcharts.

14. Cintra IP, Von Der Heydy MED, Schitz BAS, Francheschin SCC, Taddei JAAC, Sigulen DM. Métodos de inquéritos dietéticos. Cad Nutr 1997;13:11-23.

15. Philiphi ST, Szarfarc SC, Lattrza AR. Virtual Nutri (programa computador). Versão 1.0 for Windows. Departamento de Nutricão: Faculdade de Saúde Pública Universidade de São Paulo, 1996

16. Horlick M, Wang J, Pierson RN, Thornton JC. Prediction models for evaluation of total- body bone mass with dual-energy x-ray absorptiometry among children and adolescents. Pediatrics 2004; 114:337-45.

17. Fonseca JS, Martins GA. Curso de estatística. $6^{a}$ ed. São Paulo: Atlas, 1996.

18. Silva, CC, Goldberg TBL, Teixeira AS, Dalmas JC. Utilização da análise de regressão múltipla na estruturação de modelos de predição da densidade mineral óssea em adolescentes brasileiros. In: Resumos do $11^{\circ}$ Simpósio de Estatística Aplicada à Experimentação Agronômica (SEAGRO); 2005, Londrina. pp. 1-5.

19. Marshall WA, Tanner JM. Variation in the pattern of pubertal changes in boy. Arch Dis Child 1970;45:13.

20. Greulich WW, Pyle SI. Radiographic atlas of skeletal development of the hand and wrist. $2^{\text {nd }}$ ed. Stanford: University Press, 1959.

21. Sociedade Brasileira de Densitometria Clínica 2003 Consenso Brasileiro em Densitometria Clínica (online). São Paulo; 2004. Disponivel em: http://www.sbdens.org.br/intranet/publicacoes/ arquivos/Consenso2004\%203\%20Maio\%202004.pdf

22. Khan K, Mckay H, Kannus P, Bailey D, Wark J, Bennell K. Physical activity and bone health. Champaign: Human Kinetics, 2001. p.31.

23. Pessoa JHL, Lewin S, Longui CA, Mendonça BB, Bianco AC. Densidade mineral óssea: correlação com peso corporal, estatura, idade óssea e fator de crescimento símile à insulina. J Ped 1997;73:259-64.

24. Lima F, Falco V, Baima J, Carazzato JG, Pereira RMR. Effect of impact load and active load on bone metabolism and body composition of adolescent athletes. Med Sci Sports Exercise 2001;33:1318-23.

25. Warner JT, Cowan FJ, Dunstan FD, Evans WD, Webb DK, Gregory JW. Measured and predicted bone mineral content in healthy boys and girls aged 6-18 years: Adjustment for body size and puberty. Acta Paediatr 1998:87:244-9

26. Silva CC, Teixeira AS, Goldberg TBL. Impacto da ingestão de cálcio sobre a mineralização óssea de adolescentes. Rev Nutr 2004;17:351-9.

27. Rogol AD, Clark PA, Roemmich JN. Growth and pubertal development in children and adolescents: Effects of diet and physical activity. Am J Clin Nutr 2000;72(suppl): $521-8$
28. Rubin K, Schirduan V, Gendreau P, Sarfarazi M, Mendola $R$, Daisky $G$. Predictors of axial and peripheral bone mineral density in health children and adolescents, with special attention to the role of puberty. J Pediatric 1993: 123:863-70.

29. Fonseca ASM, Szejnfeld VL, Terreri MT, Goldenberg J, Ferraz MB, Hilário MOE. Bone mineralization density of the lumbar spine of Brazilian children and adolescents aged 6 to 14 years. Braz J Med Biol Res 2001;34:347-52.

30. Klein KO, Larmore KA, Lancey $\mathrm{E}$, Brown JM, Considine RV, Hassink SG. Effect of obesity on estradiol level, and its relationship to leptine, bone maturation, and bone mineral density in children. J Clin Endocrinolol Metabol 1998:83:3469-75.

31. Albuquerque MFM, Monteiro AM. Ingestão de alimentos e adequação de nutrientes no final da infância. Rev Nutr 2002;15:291-9.

32. Garcia GCB, Gambardella AMD, Frutuoso MFP. Estado nutricional e consumo alimentar de adolescentes de um centro de juventude da cidade de São Paulo. Rev Nutr 2003; 16:41-50.

33. Jackman LA, Millane SS, Martin BR, Wood OB, McCabe $G P$, Peacock $M$, et al. Calcium retention in relation to calcium intake and postmenarcheal age in adolescent females. Am J Clin Nutr 1997;66:327-33.

34. Hannan WJ, Cowen SJ, Wrate RM, Barton J. Improved prediction of bone mineral content and density. Arch Dis Child 1995;72:147-9.

35. Roemmich JN, Clarck PA, Mantzoros CS, Gurgol CM, Weltman A, Rogol AL. Relationship of leptin to bone mineralization in children and adolescents. J Clin Endocrinol Metabol 2003;88:599-604.

36. Fonseca ASM, Szejfeld VL, Fisberg $M$, Vitolo $M$, Hilário MO. Massa óssea em adolescentes obesos. Rev Bras Reumatol 1996;36:334.

37. Silva CC, Teixeira AS, Goldberg TBL. O esporte e suas implicacões na saúde óssea de atletas adolescentes. Rev Bras Med Esporte 2003:9:426-32.

38. Carvalho DCL, Rosin GC, Gama LOR, Tavares MR, Tribioli RA, Santos IR, et al. Tratamento não farmacológico na estimulação da osteogênese. Rev Saúde Pública 2002;36:647-54.

39. Goulding A, Taylor RW, Jones IE, Manning PJ, Williams SM. Spinal overload: A concern for obese children and adolescents? Osteoporos Int 2002; 13:835-40.

40. Fonseca ASMF, Terreri MT, Szejnfeld VL, Goldenberg J, Pinheiro MM, Hilário MOE. Vertebral area as a predictor of bone mass in children and adolescents. Rev Bras Reumatol 2002;42:99-103.

\section{Endereço para correspondência:}

Tamara Beres Lederer Goldberg

Departamento de Pediatria

Disciplina de Medicina do Adolescente

18607-918 Botucatu, SP

E-mail: tamara@fmb.unesp.br 